\title{
Designing Sound for a Pervasive Mobile Game
}

\author{
Inger Ekman*, Jussi Lahti, Jani Nummela, Petri Lankoski, Frans Mäyrä \\ Hypermedia Laboratory \\ University of Tampere \\ 33014 University of Tampere \\ $+35832157813^{*}$ \\ inger.ekman@uta.fi*,jussi.lahti@uta.fi,jani.nummela@uta.fi,petri.lankoski@uta.fi, \\ frans.mayra@uta.fi
}

\begin{abstract}
We examine the role of sound design in designing pervasive mobile games. As a case study, we examine the sound design process and solutions of a working prototype game called The Songs of North. The goal has been a design that allows sounds to be used as a primary information channel facilitating the use of physical movement as a main game mechanics. We discuss insights from the sound design process and also generally consider the role of sound in producing immersive gaming experiences.
\end{abstract}

\section{Keywords}

Mobile games, pervasive gaming, sound design

\section{INTRODUCTION}

Traditionally games are often thought of as a dominantly visual medium, and sound usually has only a marginal role in the game design and development process. Although there are examples of ingenious use of sound in mainstream games (for example the Thief series by Eidos), only a few games give sound a central function in play functionality; many games are even fully playable without sound. The same cannot be said for the opposite; and games rely heavily on visual information, often making it necessary to constantly watch the screen while playing.

Recently, the development of mobile phones as gaming platforms has opened new opportunities for novel game types. Whereas there has long existed handheld gaming devices (e.g. the GameBoy), combining gaming with the communication possibilities of mobile phones has opened a door to new applications, such as location-aware multiplayer games. The fact that one has to consider when developing games for such platforms is the limited display capability of such game devices. Also, there are several situations in which looking at a mobile device's screen may be impossible or unwanted, such as when driving a car or walking in a highly trafficated area. By limiting the interaction to situations in which the player is stationary, one is also setting a limit on the modes of gameplay; e.g. excluding games in which a player's movements in the physical world are a crucial aspect of playing. However, when the visual capability of a person is limited (you can only look at a few things at the time and looking at your gaming device will probably interfere with your looking at the street), the auditory channel

* Corresponding author

Proceedings of DiGRA 2005 Conference: Changing Views - Worlds in Play.

(C) 2005 Authors \& Digital Games Research Association DiGRA. Personal and educational classroom use of this paper is allowed, commercial use requires specific permission from the author. 
can still be used for conveying information about the game; we can disregard something in the audio landscape by not paying attention to it, which is what we usually do with muffled conversations in café tables. On the other hand, when necessary, we can also focus our attention towards a certain sound and really listen to it to extract the information that is being laid out for our ears to hear.

This article examines the sound design process for a pervasive mobile game (Songs of North) that is being developed as a research prototype to highlight the possibilities and design issues in this game development area. The purpose is to identify what elements of sound design can be used to support the different aspects of playability of a location-aware game that is being played on a mobile phone. The article builds on ongoing research done at the Hypermedia Laboratory at the University of Tampere. The structure of the paper is as follows: First we take a brief look at related research in the areas of game sound and mobile audio. We then describe our prototype game, The Songs of North, and the design and implementation of the game sounds and how the purpose of the game affected the design choices made. We conclude with a discussion on how the role of sound may change in mobile games and propose some directions for future work.

\section{RELATED RESEARCH}

To date, there has been remarkably little research on game audio. Most often audio solutions for gaming are searched for in earnest only when designing games for the blind and visually impaired; see e.g. $[4,5,13]$. Thus, the motivation for much audio game development has been accessibility (see e.g. the IGDA accessibility whitepaper [7]) rather than a realization of sounds potential for richer interaction. However, a few approaches have also considered sound as an alternative form of interaction for sighted players as well $[3,6,11]$.

The recent development of mobile phones as gaming platforms may call for a change in this attitude and highlight the importance of sound as offering novel opportunities from an interface design perspective. Limitations on display capability of mobile devices restrict the games visual expression, and using non-speech sound has been suggested to be the solution for making these user interfaces easier to navigate. Research on mobile sound, on the other hand, has focused on non-gaming applications. The Sonic City project [10] explored mobile interaction and wearable technology as a tool for music creation. Sonic City is a wearable music system, which creates a personal soundscape based on physical movement, local activity and urban ambiance. In another project, Nomadic Radio, different information sources received by the mobile device are combined to construct a dynamic information experience to the user [12].

\section{THE SONGS OF NORTH: A CASE STUDY}

The Songs of North is an enhanced-reality multiplayer game. The spirit world exists in parallel with the physical world. To transport the spirit to a certain place in the spirit world, players have to move around in the physical world. The game receives the movements of players through GSM cell positioning. [9] A schematic overview of the game world is presented in Figure 1.

Players act as shamans fulfilling quests in the game by gathering and manipulating objects and interacting with real and virtual characters. Interaction occurs via a virtual shaman drum (implemented in the mobile interface). The interaction between players and non-player characters can take the form of fights or collaboration. The game also supports messaging between players. 


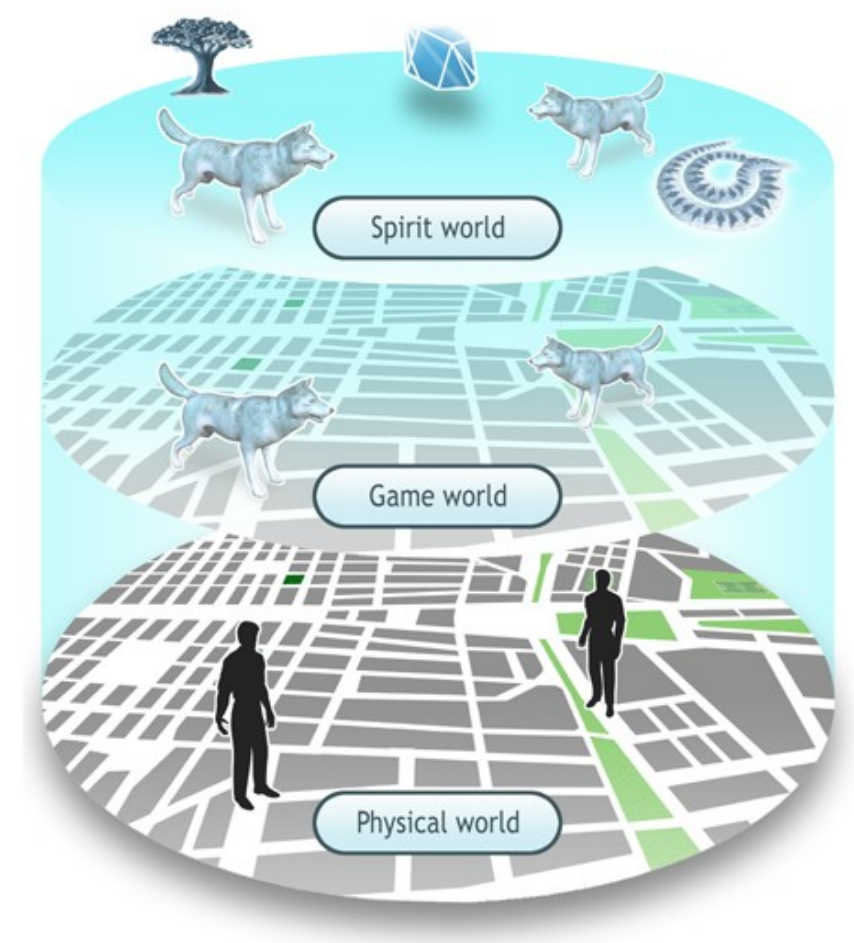

Figure 1: An overview of the enhanced-reality game world. The shaman spirit world of the game exists in parallel with the real world. Players' movements are fed into the game system depending on their location in the physical world.

\section{Sound Design in The Songs of North}

One of the main goals for the sound design of The Songs of North is to support playing without watching the game device. Because this, sound should provide enough information for some decision making in the game. With information as audio, players can move safely and monitor the location of their spirits and events in the game world. We also wanted to provide strong auditory support for visually perceivable events, to heighten the sense of an immersive game world. The moments when the player looks at the visual information simultaneously with hearing audio can also act as learning points introducing audio as a primary information channel.

The basic idea in the audiovisual design of the game is that the spirit world can be heard realtime, whereas getting up-to-date visual information requires casting spells with a shaman drum (mobile device). This way, audio is 'free' in the sense that it doesn't require the player to do anything. Since information is available visually as well, players can gradually shift to using audio information as they realize the benefits of movement for playing. A listener can also process audio in the background and notice changes in sounds even if they were not directly attending to them [2]. This way, since audio is not the only channel of information, sound can be used as a compromise between active playing and not attending to the game at all. Thus, when players notice something requiring their attention, they can pick up their shaman drum and require visual information. This design is intended to encourage players to use the audio information as an event signal even before they know exactly what a particular sound means, and 
thus support physical movement as a main game mechanic from the very beginning of the game.

Game sounds are used to convey information of places, objects and characters nearby as well as movement or other actions of characters within a certain range. Characters, places and game objects have specific sounds, which are played depending on their range from the player. As the players move in the real world, or as NPCs move within the game world, the distances change. Movements are reflected in the soundscape as addition or deletion of sounds.

There were seven types of sounds to be played for a player:

- Player's own drum hit sounds

- Drum hit pattern sounds for other players' actions

- Character sounds

- $\quad$ Item sounds for different item classes

- Place sounds either for unique places or place types in the game world

- $\quad$ Attack sounds for different attack types (or elements)

- Ambient sounds that represent the world's current state (good, neutral or evil)

The Java-implementation for Nokia N-Gage (our test device) did not support playing several sounds at once. Because of this, sounds had to be queued in order of play priority and played when possible. Since the drum is the player's primary tool for manipulating the game world, a design decision was made to give the topmost priority to the player's own drum hits. Whenever a player uses the drum to make a spell, all other game sounds quiet down and the drum sound is played.

All other sounds are queued and played one by one. Every time the game device observes the game world (at least once every 10 seconds), the game world is examined to decide, what objects, characters and places are presently in the proximity of the character. The sounds of these, as well as the state sound describing the nature of the game world are queued in chronological order, making the most recent sound last in the queue. The maximum number of sounds in the queue at once is eight and too old sounds are deleted from the queue as newer ones arrive. The same sound can appear in the queue only once, thus a sound is not added if the queue already holds one prior instance of it. Sounds are popped and played from the queue with a random amount of silence between them. Additionally, to avoid unnecessary repetition, the ambient sound (representing the game world's current state), which would otherwise always be in the queue, was played only once every three minutes.

To make the soundscape more diverse, characters could produce several different sounds. Character sounds were either specific or generic. The specific character sound was a sonic symbol for the type and current state of the character (indication of totem animal, modified according to three possible character states: attack, damage and normal). Alternatively the character's presence was conveyed via a generic footstep sound. Sometimes characters did not 
produce sounds at all. The probabilities for choosing the character sound are: 0.5 for the generic movement sound, 0.33 for character class/state and 0.17 for silence.

In order to make the identification of sounds as easy as possible, we chose to use very realistic sounds to depict events and characters (footsteps for walking, the sounds of a howling wolf for wolf-shamans etc.). An alternative would have been to use more abstract sound, such as earcons (see e.g. [1]). Research on different types of sonic identifiers has shown that auditory icons are more readily learned and remembered than abstract sound [8]. Moreover, the use of more realistic sounds for game objects and events was not only based on the recognition of sounds, but also a stylistic choice to lend the game world a soundscape that could be perceived as a shaman spirit world.

\section{Emergent Issues in Sound Design for Mobile Games}

The first and foremost issue we had to deal with when making the sound design and implementation was the technological limitations of the mobile device. Technically, such issues as limited storage and the low-level controllability of sound somewhat tie the hands of designers if they are accustomed to the solutions available in pc or console gaming. Moreover, the sound quality of the mobile devices is often optimized for speech, and may not scale very well for all purposes.

Another issue worth considering is the difference in listening situation compared to a traditional stationary gaming setting. The usual speaker settings that are part of home systems will often have some spatial sound available (at least a left/right distinction), but this is not guaranteed with mobile games Although headphones are available, it is uncertain whether people will use them or rather have the phone in their pocket. If sounds are played on the speakers of the phone, they do not necessarily serve to immerse players in the game world, but may instead highlight the presence of the game device at the expense of immersion. Also, with enhanced-reality mobile gaming, spatial sound cues require a knowledge of which way the player is headed, which sets the demand for special technology such as, for example, a compass integrated into headphones with feedback to the game device. Furthermore, bringing gaming out of the living room will mean that the sound design has to take into account all sorts of noises, which may affect the intelligibility of sounds relevant for the player. In a mobile gaming situation, the sound has to deal with the various sound contexts of everyday life. Background noise, conversations and other activities may affect the intelligibility of game audio or mask the sounds so that they cannot be heard.

Also something worth consideration is the social playability of mobile games. Whereas sound design for console and PC games could concentrate just on making a brilliant soundscape, social playability is an important issue in mobile game sound design. This is an issue that is rather unique for mobile games. Sound design for mobile devices has to consider also how other people, not at all engaged with playing, will relate to the soundscape. For example, the ideal volume of the sounds may vary depending on the situation a player is in, not only providing maximum intelligibility, but social acceptability as well. Social playability also means considering the gaming context in a broader sense. This involves designing the game interaction as a whole in such a way that it fits in with the players activities. This is not to imply that games should not try to change players' attitudes. However, what we argue is that when designing games to be played in a social context, sound design (as well as the design of any other 
information channel) should not be seen as independent design tasks. For example, we have argued that the use of sound can be used as a means to minimize the need for visual interaction with the device. In addition to being essential to play functionality (allowing the players to use movement as a game mechanics), this also means that it is possible for a player to most of the time play unnoticed by others. This may be a crucial factor for some player groups, if they do not want to be recognized as players when out in the city.

The notion of social playability can also be thought of as connected with a larger shift in playing habits. What mobile is bringing to gaming is the ability for people to engage in play wherever they are - at a bus stop, in the workplace cafeteria, on the train. Together with the communication possibilities of mobile devices, there is an underlying potential for a shift in how we look at the whole (computer) gaming experience: from a solitary and foremost private activity towards social interaction within a gaming context. With time, it is probable also that attitudes towards gaming in a social context changes and games and gaming (and, also, game sounds) are seen as a normal part of the everyday activities of people.

As a final note, we view enhanced reality gaming not only as providing dizzying gaming experiences. Ultimately, we think that the combination of moving around in the physical world in parallel with the sense of moving around in a fictional world, where the player has the ability to manipulate and affect the environment, could enhance the players' experience of their everyday surroundings. Successful design for immersion may encourage players to view the physical world with a new attitude. Eventually, the game world might well add color and meaning to our everyday events, making a trip to the supermarket a challenging endeavor into a fierce battleground or walking the dog a trip to the treasury.

\section{CONCLUSIONS AND FUTURE WORK}

We have examined the role of sound design in a mobile pervasive game in a prototype mobile game called The Songs of North. The work presented here is part of ongoing research done at the Hypermedia Laboratory at the University of Tampere. In the next stage of research, we will continue to develop the game prototype as well as perform user tests on the sound design and implementation. One task is to make the audiovisual integration stronger, and more profoundly define the roles the visual and audio components play in the interface.

Another interesting question related to this is what different roles sound can play in the interface. The research described in this paper examined the use of sound to provide real-time task-oriented information on events, objects, characters and places within proximity of the player in the game world. In the future we will examine how (or, indeed if) sound can be used in other roles as well, such as providing background information on the game world or to lend emotional impact to events and places in the game world.

\section{AKNOWLEDGEMENTS}

We thank Satu Heliö for doing a tremendous job with the quest and story design in The Songs of North. The project is funded by National Technology Agency TEKES, Codetoys, the Finnish national lottery Veikkaus, Nokia Research Center and TeliaSonera Finland.

\section{REFERENCES}

1 Blattner, M., Sumikawa, D. and Greenberg, R. Earcons and icons: Their structure and common design principles. Human Computer Interaction, 4, 1 (1989), 11-44. 
Cohen, J. Monitoring background activities. In Kramer, G. (ed.) Auditory Display. SFI Studies in the Sciences of Complexity. Addison-Wesley, 1994.

3 Drewes, T.; Mynatt, E. \& Gandy, M. Sleuth: An Audio Experience. Proc. International Conference on Auditory Display. Atlanta, GA, USA, 2000.

4 Eriksson, Y \& Gärdenfors, D. Computer Games for Children with Visual Impairments. Proc. 5th International Conference of Disability, Virtual Reality \& Associated Technologies, Oxford, UK, 2004, 79-86.

5 Friberg, J. \& Gärdenfors, D. Audio Games: New Perspectives on Game Audio. Proc. Advances in Computer Entertainment Technology. Singapore, 2004.

6 Hadrup, R., Jakobsen, P. S., Juul, M. S., Lings, D., Magnúsdóttir, Å., O. Designing an Auditory WLAN based Game. 2004. http://www2.hku.nl/ audiogam/ag/articles/Designing\%20an\%20Auditory\%20WLAN\%20based\%20Game.pdf [Accessed February 15, 2005.]

7 IGDA. Accessibility in Games: Motivations and Approaches. International Game Developers Association white paper. 2004. http://www.igda.org/accessibility/IGDA_Accessibility_WhitePaper.pdf [Accessed February 15, 2005.]

8 Keller, P. \& Stevens, C. (2004). Meaning From Environmental Sounds: Types of Signal-Referent Relations and Their Effect on Recognizing Auditory Icons. Journal of Experimental Psychology: Applied, 10, 1 (2004), 3-12.

9 Lankoski, P.; Heliö, S.; Nummela, J.; Lahti, J.; Mäyrä, F.; Ermi, L. (2004). A Case Study in Pervasive Game Design: The Songs of North. Proc. NordiCHI, Tampere, Finland, 2004, 413-416.

10 Mazé, R. \& Jacobs, M. Sonic City: Prototyping a Wearable Experience. Proc. 7th IEEE International Symposium on Wearable Computing. 2003.

11 McCrindle, R. J. \& Symons, D. Audio Space Invaders. Proceedings of the 3rd International Conference of Disability, Virtual Reality \& Associated Technologies, Alghero, Italy, 2000, 59-65.

12 Sawhney, N. \& Schmandt, C. Nomadic Radio: Speech and Audio Interaction for Contextual Messaging in Nomadic Environments. ACM Transactions on Computer-Human Interaction, 7, 3 (2000), 353-383.

13 Targett, S. \& Fernström, M. Audio Games: Fun for All? All for Fun? Proc. International Conference on Auditory Display, Boston, MA, USA, 2003. 